\title{
SPORADIC RE-EMERGENCE OF ENZOOTIC PORCINE TRANSMISSIBLE GASTROENTERITIS IN HUNGARY
}

\author{
Márta LÖRINCZ ${ }^{1}$, Imre BIKSI ${ }^{2}$, Simon ANDERSSON ${ }^{1}$, Attila CSÁGOLA $^{1}$ \\ and Tamás TUBOLY ${ }^{1 *}$ \\ ${ }^{1}$ Department of Microbiology and Infectious Diseases, Faculty of Veterinary Science, \\ Szent István University, Hungária krt. 23-25, H-1143 Budapest, Hungary; \\ ${ }^{2}$ Large Animal Clinic, Faculty of Veterinary Science, Szent István University, \\ Dóra major, Üllö, Hungary
}

(Received 9 January 2013; accepted 2 April 2013)

Transmissible gastroenteritis (TGE) is a coronavirus-induced disease of pigs, characterised by diarrhoea and vomiting. The incidence of the disease had been decreasing since the late 1980s when deletion mutant variants (porcine respiratory coronavirus, $\mathrm{PRCoV}$ ) of the virus emerged, repressing TGE gradually. Although disease manifestations are infrequent, the virus is still present in pig herds, causing sporadic outbreaks in a milder form. Identification and characterisation of the spike genes from TGEV and PRCoV, detected in such outbreaks, were performed in Hungary. Analysis of the amplified partial gene sequences showed that TGEV was present in herds with TGE clinical signs together with PRCoV. The sequences, apart from the deletions in $\mathrm{PRCoV}$, were identical and at least two types of PRCoV spike proteins could be identified based on the length of the deleted sequence.

Key words: Transmissible gastroenteritis, coronavirus, swine diarrhoea

Porcine transmissible gastroenteritis (TGE) is caused by a coronavirus belonging into the Alphacoronavirus genus of the Coronaviridae family (de Groot et al., 2012). Coronaviruses are enveloped and contain the largest known singlestranded viral RNA genome of positive polarity (26-32 kilobases, kb). TGE was first identified by Doyle and Hutchings (1946) and caused severe economic problems in the pig industry until the late 1980 s, characterised by vomiting, diarrhoea and dehydration (Hooper and Haelterman, 1969) in younger pigs. Pigs infected later in life may not even show clinical signs of the infection (Moon et al., 1973). In Belgium, there was a dramatic increase in animals being seropositive for TGEV in 1984 without clinical signs associated with TGEV infection (Pensaert et al., 1986). TGEV could not be isolated from the seropositive animals; instead, a new mutant of TGEV (porcine respiratory coronavirus, PRCoV) charac-

*Corresponding author; E-mail: Tuboly.Tamas@aotk.szie.hu; Phone: 0036 (1) 251-9900; Fax: 0036 (1) 251-9260 
terised mainly by deletions in the spike (S) protein (Rasschaert et al., 1990; Britton et al., 1991) emerged. PRCoV is pneumotropic, replicating mainly in alveolar cells, but it can also replicate in the gastrointestinal tract in cells located underneath the villi, and the infections are usually inapparent (Cox et al., 1990).

There is a strong antigenic connection between TGEV and PRCoV. Sows infected with PRCoV secrete antibodies with the milk that are capable of decreasing the infection rate in the newborn gut, reducing clinical manifestation and consequently mortality of TGE (De Diego et al., 1994). Protective immunity on the antibody level against TGE is directed to the S protein of the virion. The spike is responsible for the attachment of the virus to cellular receptors. The major such receptor is the ubiquitous aminopeptidase-N (Delmas et al., 1992) used both by TGEV and PRCoV, but another intestinal receptor for TGEV was suggested by Weingartl and Derbyshire (1994) that can explain the age-limited susceptibility of pigs and the differences in pathogenicity of TGEV and PRCoV. One of the differences between TGEV and PRCoV is the deletion of variable size in the S gene (up to 681 nucleotides, Kim et al., 2000) that affects the intestinal binding of the virus either through the suggested receptor or by lacking the ability to utilise sialic acid when penetrating the mucus layer of the small intestine. The same deletion is suitable for the differentiation of TGEV and PRCoV by molecular diagnostic techniques (Paton et al., 1997) or by serology (Simkins et al., 1992).

With the appearance and spread of PRCoV, the incidence of TGE gradually decreased. However, occasional reports of TGEV-specific seropositivity (Elvander et al., 2000; Brendtsson et al., 2006; Roic et al., 2012) and even sporadic outbreaks in different parts of the world (Pritchard et al., 1999; Kim et al., 2000; Miyazaki et al., 2010; Wang et al., 2010) indicate that TGE cannot be neglected, as the virus is still present in pig herds, although mostly without clinical manifestations.

Recently, disease outbreaks characterised by vomiting and diarrhoea of 57 days old suckling piglets were observed on a large sow farm in Hungary. Affected litters were mainly those of gilts, but litters of some older sows showed similar clinical signs. These usually started with vomiting, then liquid, yellowish diarrhoea was seen, and emaciation, dehydration of the piglets developed rapidly. The disease spread rapidly to younger piglets in the room. Morbidity in some groups was estimated at about $50 \%$, while mortality stayed low, at about 4-6\%. Sows were in good general condition, and liveborn litter sizes and piglet birth weights were within acceptable limits. The farm was run according to strict hygienic standards. It was not possible to relate outbreaks of vomiting and diarrhoea to sow feed composition or to particular farrowing rooms. The problem was more frequently observed from late autumn to early spring. Other age groups on the farm did not experience a similar condition. The herd was free of the main infectious agents including PRRSV, Aujeszky's disease virus, $M y$ coplasma hyopneumoniae, Actinobacillus pleuropneumoniae serotypes 1 and 2, and Brachyspira hyodysenteriae. 
The purpose of the present study was to screen the affected animals for the presence of coronaviruses (PRCoV and TGEV), and to characterise the potential differences in the spike gene sequences of these viruses.

\section{Materials and methods}

Samples

Four clinically affected, untreated piglets of 4-6 days of age were sacrificed and dissected on the farm. Samples were collected from the gastrointestinal tract and parenchymal organs for histopathology and immunohistochemistry (not shown). Samples for aerobic bacteriological culture, detection of clostridia and other common pathogenic anaerobic bacteria and viruses were also collected from the small and large intestines. After the detection of the first TGEVpositive cases, a wider survey was initiated, where samples of small intestines, faeces and lung tissues were collected from 150 animals of the most susceptible age group. These samples were gathered at 14 different farms throughout the country, including the original farm. All of the farms were infected with porcine circovirus type 2 (data not shown).

At the farm where the first TGEV-positive piglets were found, blood samples intended for serological examination were drawn from 15 gilts and 15 multiparous sows nursing affected litters. These samples were tested for the presence of anti-TGEV antibodies with a TGEV/PRCoV differentiating commercial ELISA (Svanovir ${ }^{\circledR}$ TGEV/PRCV-Ab ELISA, Svanova, Sweden) as described by the manual.

\section{Reverse transcriptase polymerase chain reaction (RT-PCR) and cloning}

About $0.1 \mathrm{~g}$ of the tissues or the faecal samples was used for nucleic acid extraction with the Viral Gene-spin ${ }^{\mathrm{TM}}$ Viral DNA/RNA Extraction Kit (iNtRON Biotechnology Inc., Korea) as recommended by the manufacturer using $0.5 \mu 1$ of $20 \mathrm{mg} / \mathrm{ml}$ Proteinase K (Fermentas, Lithuania). Reverse transcription of the RNA templates was performed with the M-MuLV RevertAid ${ }^{\mathrm{TM}}$ Reverse Transcriptase using random hexamer primers (100 pmol/sample) (Fermentas, Lithuania).

The PCR primers used for the amplification and differentiation of the $\mathrm{S}$ gene from TGEV or PRCoV were designed using the Primer 3 program (Rozen and Skaletsky, 2000) based on the TGEV Purdue 115 full genome sequence (GenBank Accession Number: DQ811788), where the S gene is located at 20354-24697 nucleotides (nt). The primers were TGE2: 5'-TAAGGAAGGGTAAGTTGCTCA-3' (binding at 20282-20301 nt) and TGE3: 5'-TGGTCCATCAGTTACGCCGAA-3' (21538-21518 nt) flanking a 1218-base fragment of the TGEV-S gene, covering the usual deletion sites of PRCoV-S. The reaction mixtures contained $1 \mu \mathrm{cDNA}, 1 \mu \mathrm{l}$ of each primer ( $25 \mathrm{pmol}), 1 \mu \mathrm{l}$ of dNTP $(1 \mathrm{mmol}), 1$ unit DreamTaq DNA poly- 
merase (Fermentas, Lithuania) and $5 \mu$ of 10x DreamTaq Buffer (Fermentas, Lithuania) in a final volume of $50 \mu$ l. The amplification was done in a TGradient Thermocycler (Biometra, Germany). Molecularly cloned TGEV-S gene (Tuboly et al., 1994) was used as positive control.

The steps of the PCR were as follows: 5 min at $95^{\circ} \mathrm{C}$, followed by $40 \mathrm{cy}-$ cles of denaturation at $95^{\circ} \mathrm{C}$, annealing at $55^{\circ} \mathrm{C}$ (both for $30 \mathrm{sec}$ ) and elongation at $72{ }^{\circ} \mathrm{C}$ for $1 \mathrm{~min}$, with a final step at $72{ }^{\circ} \mathrm{C}$ for $7 \mathrm{~min}$. The amplicons were analysed in $2 \%$ agarose gels by electrophoresis. PCR fragments of sizes characteristic of TGEV- or PRCoV-S genes were molecularly cloned using the TOPO ${ }^{\circledR}$ TA Cloning ${ }^{\circledR}$ Kit (Invitrogen, USA) following the instructions of the manufacturer.

\section{Sequence analysis}

The purified DNA clones were sequenced at the Biomi Ltd. (Gödöllö, Hungary) using the Big Dye ${ }^{\circledR}$ DirectCycle Sequencing Kit (Invitrogen, USA), with the universal M13 reverse and forward primers, in an ABI310 automated sequencer. Sequences were edited and aligned using the BioEdit (Hall, 1999) and MEGA5 software products (Tamura et al., 2011), and compared to known TGEV and PRCoV S gene sequences of the GenBank.

\section{Results}

\section{Pathology and histopathology}

Gross pathological lesions, found in piglets that succumbed to the disease or had to be euthanised due to terminal illness, were weight loss and signs of dehydration. The distended small intestinal loops and large intestines were filled with yellowish fluid. Mesocolonic oedema also occurred. Histopathology of the small intestinal tract did not reveal major changes apart from mild shortening of the villi. Crypt hyperplasia was not evident in the cases examined and the colonic mucosa appeared normal. Parenchymal organs did not show pathognomonic alterations. Common pathogens (pathogenic Escherichia coli, Clostridium perfringens C) were not detectable in any of the samples, while others (Clostridium perfringens A, Clostridium difficile $\mathrm{A} / \mathrm{B}$ toxins) were detected in a small subset of these samples.

\section{Reverse transcriptase polymerase chain reaction}

The results of the RT-PCRs indicated that out of the 150 samples originating from 14 different swine herds only four herds were TGEV positive. Seven herds were positive for PRCoV only and three were negative both for TGEV and PRCoV. The farms could be divided into three groups based on their TGEV/ PRCoV profiles. 
In the first group, pigs that suffered from clinical signs associated with enzootic TGE usually had either PRCoV- or TGEV-S gene sequences in their samples. These gene fragments were estimated by electrophoresis to be 550 base pairs (bp) and $1250 \mathrm{bp}$, for PRCoV- and TGEV-S genes, respectively. Mixed infections were rarely detected. The PRCoV-S gene fragments were uniform in size.

The second group also consisted of animals showing clinical signs of enzootic TGEV infection but in this group $\mathrm{S}$ genes of a variety of sizes were detected. Besides the full-size fragment of the original TGEV-S (1258 bp, confirmed by sequencing), a variety of smaller-sized fragments were also detected, the dominant among them being a fragment with an approximate size of $600 \mathrm{bp}$ and another of $250 \mathrm{bp}$, as judged by agarose gel electrophoresis. These three fragments were selected for cloning and sequencing. Besides these three $\mathrm{S}$ gene variants, others of intermediate sizes were also detected, but the cloning of these minor fragments was unsuccessful, or the obtained sequence did not show any homology with coronavirus genomes (data not shown). The original full-size fragment of the TGEV-S was found together with the smaller fragments in the same animal, which sets this group apart from the first one.

Animals of the third group did not show clinical signs of enzootic TGEV infection. On these farms the TGEV-S gene was not detected. The S gene amplicons were of approximately 550-600 bp in length, consistent with what would be expected in PRCoV-positive animals, but smaller fragments were also present.

When comparing the presence of TGEV and PRCoV in different organs such as the small intestine, lungs or lymph nodes, no difference could be observed. Usually lung and gut tissues or intestinal contents carried the virus. Hence, organ preference or tropism could not be established.

\section{Sequence analysis}

The largest fragment was 1258 bp as expected (GenBank No.: KC800687), the middle one had a size of $586 \mathrm{bp}$ (GenBank No.: KC800688), while the shortest $\mathrm{S}$ gene amplicon was 283 bp in length.

The GenBank search of these nucleotide sequences showed that they were 98-99\% identical to the viruses of the PUR46-MAD type (Sanchez et al., 1992) and fully identical on the predicted amino acid level with the corresponding area of the Chinese virulent field strain WH-1 (ADY39740). When comparing the amplicons to one another, it was obvious that the same coronavirus existed in at least three different forms if looking only at the S gene. Namely, the full-length TGEV-S type, a shorter one with a $672 \mathrm{nt}$ deletion at the amino terminal half coding region of the $\mathrm{S}$ gene, and a further deletion mutant where the deletion was extended with an additional 303 nt towards the 3' end of the gene. Still, all of these variants carried the sequence characteristics of the TGEV identified during this study, when only looking at the non-deleted parts of the gene. All of these 
deletions retained the functionality of the remaining portion of the $\mathrm{S}$ protein, as the number of nucleotides missing did not alter the reading frame of the gene.

\section{Serology}

The results are summarised in Table 1, where the ELISA positivity of pigs from the original TGEV cases is shown for both TGEV and PRCoV. The majority of the gilts were seropositive for porcine coronavirus and 6 out of 11 animals showed TGEV positivity at the threshold level. The older sows all seroconverted against porcine coronavirus and only 2 out of the 15 animals tested were seronegative for TGEV.

Table 1

ELISA results from blood samples collected from 15 gilts and 15 older sows in a TGEV-PRCoV differentiating test

\begin{tabular}{lcccccc}
\hline & \multicolumn{3}{c}{ TGEV } & \multicolumn{3}{c}{ PRCoV } \\
\hline Gilts & Positive & Suspect & Negative & Positive & Suspect & Negative \\
No. & 0 & 6 & 9 & 11 & 0 & 4 \\
$\%$ & 0 & 40 & 60 & 73.3 & 0 & 26.67 \\
\hline Older sows & Positive & Suspect & Negative & Positive & Suspect & Negative \\
No. & 5 & 8 & 2 & 15 & 0 & 0 \\
$\%$ & 33.33 & 53.33 & 13.33 & 100 & 0 & 0 \\
\hline
\end{tabular}

\section{Discussion}

The first appearance of transmissible gastroenteritis (Doyle and Hutchings, 1946), when pig herds without any immunity encountered the virus, was devastating. This epizootic form of the disease was typical in newly affected herds, with $100 \%$ mortality rate of newborn animals. In older animals the clinical signs were restricted to mild diarrhoea and decreased production. As the epizootic proceeded worldwide and more and more herds seroconverted, the mortality rate dropped to $10-50 \%$ in newborns, depending on maternal antibody levels. Still, even with its sporadic epizootic and characteristic enzootic form TGE was one of the major viral diseases of the swine industry until the appearance and gradual spread of the mutant PRCoV strains. The origin of PRCoV is not known; it was suspected that some attenuated TGEV vaccine started an individual spread worldwide, but the sequence differences and the dissimilarities in the site and size of deletions among the identified strains indicated otherwise (Tuboly, 1996).

Although the PRCoV-induced antibodies provide only limited protection against TGEV, the constant presence of the new virus and the PRCoV antibodies resulted in a decrease of the incidence of clinical TGE in PRCoV-infected herds. By the mid-1990s only sporadic cases of TGE were reported. Today TGE is usu- 
ally considered as a disease of the past. However, there have been occasional reports of TGEV seropositivity (Elvander et al., 2000; Brendtsson et al., 2006), indicating that the virus was still present, but at levels below the threshold of clinical manifestation.

Based on the results of this study, the herd where clinical signs and histopathology first raised the suspicion of a TGEV infection was indeed proven to be TGEV positive, both by serological and PCR tests. In the TGEV-positive cases, mild villous atrophy was seen without any other significant gross or microscopic alteration. Group 1 coronavirus (Alphacoronavirus 1) immunohistochemistry (not shown) on small intestinal samples gave dubious results. When the survey was extended to other herds, the presence of TGEV could be confirmed with PCR in four pig farms. From the re-emergence of the disease one would expect that a new, perhaps more virulent TGEV strain is spreading, one that is capable of breaking through the immunity induced by PRCoV. However, the sequencing results indicated that the viruses were very similar to already known TGE viruses, namely to those of the PUR46-MAD strain (Sanchez et al., 1992). In order to decide if this truly is a new genetic variant of the virus with higher virulence, other regions of the genome must also be amplified and sequenced.

The PRCoV sequences detected in this study could be separated into two different groups: one with a $672 \mathrm{nt}$ deletion and one where the deletion was altogether $975 \mathrm{nt}$ long. The $672 \mathrm{nt}$ deletion (compared to the TGEV-S gene) was identical to what was observed for the PRCoV strains widespread in Europe, where a 224 amino acid (aa) coding region was deleted starting at the position of amino acid number 21 of the $\mathrm{S}$ gene. It is, however, peculiar that after several years of establishing the genetic characteristics of the PRCoV strains in Europe the same deletion mutant is still present in pig herds.

The presence of the PRCoV genomes with an even longer deletion within the $\mathrm{S}$ gene was surprising as such large deletions starting at the same site as the previous one and extending $303 \mathrm{nt}$ further into the 3' direction have not been reported previously. This raises the question whether such deletion mutants can form infectious particles, or if they can only survive when packaged into virions produced by co-existing longer genomes. The possibility that this deletion mutant remains infective is likely as the $975 \mathrm{nt}$ deletion by itself should not thwart virus infection. This is so because the aminopeptidase- $\mathrm{N}$ binding site is encoded further downstream on the sequence as compared to the end of the deletion. Without structural studies of such an S protein variant it is of course difficult to tell how the lack of such a long protein stretch may change the conformation of the receptor binding site.

Our results cannot explain the re-emergence of the virus in clinical conditions, but from this limited survey it seems that primiparous sows did not completely seroconvert to TGEV, therefore their piglets were probably not fully protected. PRCoV seronegativity was detected in a limited number of primiparous sows, indicating that cross-protection might also be suboptimal. These results in- 
dicate that the answer to why TGE is re-emerging lies not within the genetics of the virus but most likely in the immune response of the pigs. It is known that porcine circoviruses (PCV) are present worldwide and they are strongly immunosuppressive (Ramamoorthy and Meng, 2009). Vaccine- or infection-induced immunity is generally limited in heavily infected pig herds, even if the clinical signs of circovirus infection are not apparent. Although we have no direct proof of this assumption, namely that the presence of PCV is responsible for the TGEV problem, serological results (Table 1) showed that primiparous pigs may remain seronegative for both TGEV and PRCoV. Similar increases of TGE incidence have been reported from Japan (Miyazaki et al., 2010) and China (Wang et al., 2013), the world's largest pork producer.

\section{Acknowledgement}

The research studies of Dr. Attila Cságola were financed in part by the János Bolyai Research Scholarship of the Hungarian Academy of Sciences.

\section{References}

Brendtsson, L. T., Thorén, P., Escutenaire, S. and Wallgrean, P. (2006): Seroreactors to TGEV found at two different occasions in a country free from TGEV but infected with PRCV. Proceedings of the 9th IPVS Congress, Copenhagen, Denmark, volume 2, p. 145.

Britton, P., Mawditt, K. L. and Page, K.W. (1991): The cloning and sequencing of the virion protein genes from a British isolate of porcine respiratory coronavirus: comparison with transmissible gastroenteritis virus genes. Virus Res. 21, 181-198.

Cox, E., Pensaert, M. B., Callebaut, P. and van Deun, K. (1990): Intestinal replication of a porcine respiratory coronavirus closely related antigenically to the enteric transmissible gastroenteritis virus. Vet. Microbiol. 23, 237-243.

De Diego, M., Rodríguez, F., Alcaraz, C., Gómez, N., Alonso, C. and Escribano, J. M. (1994): Characterization of the IgA and subclass IgG responses to neutralizing epitopes after infection of pregnant sows with the transmissible gastroenteritis virus or the antigenically related porcine respiratory coronavirus. J. Gen. Virol. 75, 2585-2593.

de Groot, R. J., Cowley, J. A., Enjuanes, L., Faaberg, K. S., Perlman, S., Rottier, P. J. M., Snijder, E. J., Ziebuhr, J. and Gorbalenya, A. E. (2012): The order Nidovirales. In: King, A. M. Q., Adams, M. J., Carstens, E. B. and Lefkowitz, E. J. (eds) Virus Taxonomy: Classification and Nomenclature of Viruses: Ninth Report of the International Committee on Taxonomy of Viruses. Elsevier Academic Press, Amsterdam. pp. 785-795.

Delmas, B., Gelfi, J., L'Haridon, R., Vogel, L. K., Sjostrom, H., Noren, O. and Laude, H. (1992): Aminopeptidase $\mathrm{N}$ is a major receptor for the enteropathogenic coronavirus TGEV. Nature 357, 417-420.

Doyle, L. P. and Hutchings, L. M. (1946): A transmissible gastroenteritis in pigs. J. Am. Vet. Med. Assoc. 108, 257-259.

Elvander, M., Wallgren, P., Klingeborn, B. and Engvall, A. (2000): Serological surveys indicating a new antibody pattern in TGEV/PRCV serology in Sweden. Proceedings of the Fifth ESVV Conference, Brescia, Italy. pp. 285-286.

Hall, T. A. (1999): BioEdit: a user-friendly biological sequence alignment editor and analysis program for Windows 95/98/NT. Nucl. Acids Symp. Ser. 41, 95-98. 
Hooper, B. E. and Haelterman, E. O. (1969): Lesions of the gastrointestinal tract of pigs infected with transmissible gastroenteritis. Can. J. Comp. Med. 33, 29-36.

Kim, L., Hayes, J., Lewis, P., Parwani, A. V., Chang, K. O. and Saif, L. J. (2000): Molecular characterization and pathogenesis of transmissible gastroenteritis coronavirus (TGEV) and porcine respiratory coronavirus (PRCV) field isolates co-circulating in a swine herd. Arch. Virol. 145, 1133-1147.

Miyazaki, A., Fukuda, M., Kuga, K., Takagi, M. and Tsunemitsu, H. (2010): Prevalence of antibodies against transmissible gastroenteritis virus and porcine respiratory coronavirus among pigs in six regions in Japan. J. Vet. Med. Sci. 72, 943-946.

Moon, H. W., Norman, J. O. and Lambert, G. (1973): Age dependent resistance to transmissible gastroenteritis of swine (TGE) 1. Clinical signs and some mucosal dimensions in small intestine. Can. J. Comp. Med. 37, 157-166.

Paton D., Ibata, G., Sands, J. and McGoldrick, A. (1997): Detection of transmissible gastroenteritis virus by RT-PCR and differentiation from porcine respiratory coronavirus. J. Virol. Methods 66, 303-309.

Pensaert, M., Callebaut, P. and Vergote, J. (1986): Isolation of a porcine respiratory, non-enteric coronavirus related to transmissible gastroenteritis. Vet. Q. 8, 257-261.

Pritchard, G. C., Paton, D. J., Wibberley, G. and Ibata, G. (1999): Transmissible gastroenteritis and porcine epidemic diarrhoea in Britain. Vet. Rec. 144, 616-618.

Ramamoorthy, S. and Meng, X. J. (2009): Porcine circoviruses: a minuscule yet mammoth paradox. Anim. Health Res. Rev. 10, 1-20.

Rasschaert, D., Duarte, M. and Laude, H. (1990): Porcine respiratory coronavirus differs from transmissible gastroenteritis virus by a few genomic deletions. J. Gen. Virol. 71, 2599-2607.

Roic, B., Jemersic, L., Terzic, S., Keros, T., Balatinec, J. and Florijancic, T. (2012): Prevalence of antibodies to selected viral pathogens in wild boars (Sus scrofa) in Croatia in 2005-06 and 2009-10. J. Wildl. Dis. 48, 131-137.

Rozen, S. and Skaletsky, H. J. (2000): Primer3 on the WWW for general users and for biologist programmers. In: Krawetz, S. and Misener, S. (eds) Bioinformatics Methods and Protocols: Methods in Molecular Biology. Humana Press, Totowa, NJ. pp. 365-386.

Sanchez, C. M., Gebauer, F., Sune, C., Mendez, A., Dopazo, J. and Enjuanes, L. (1992): Genetic evolution and tropism of transmissible gastroenteritis coronaviruses. Virology 190, 92-105.

Simkins, R. A., Weilnau, P. A., Bias, J. and Saif, L. J. (1992): Antigenic variation among transmissible gastroenteritis virus (TGEV) and porcine respiratory coronavirus strains detected with monoclonal antibodies to the S protein of TGEV. Am. J. Vet. Res. 53, 1253-1258.

Tamura, K., Peterson, D., Peterson, N., Stecher, G., Nei, M. and Kumar, S. (2011): MEGA5: Molecular Evolutionary Genetics Analysis using maximum likelihood, evolutionary distance, and maximum parsimony methods. Mol. Biol. Evol. 28, 2731-2739.

Tuboly, T. (1996): Enteric coronaviruses of pigs (in Hungarian, with English abstract). Magyar Állatorvosok Lapja 51, 361-365.

Tuboly, T., Nagy, É., Dennis, J. R. and Derbyshire, J. B. (1994): Immunogenicity of the S protein of transmissible gastroenteritis virus expressed in baculovirus. Arch. Virol. 137, 55-67.

Wang, C., Chen, J., Shi, H., Qiu, H., Xue, F., Liu, S., Liu, C., Zhu, Y., Almazán, F., Enjuanes, L. and Feng, L. (2010): Rapid differentiation of vaccine strain and Chinese field strains of transmissible gastroenteritis virus by restriction fragment length polymorphism of the $\mathrm{N}$ gene. Virus Genes 41, 47-58.

Wang, L., Ge, C., Wang, D., Li, Y. and Hu, J. (2013): The survey of porcine teschoviruses, porcine circovirus and porcine transmissible gastroenteritis virus infecting piglets in clinical specimens in China. Trop. Anim. Health Prod. 45, 1087-1091.

Weingartl, H. and Derbyshire, J. B. (1994): Evidence for a putative second receptor for porcine transmissible gastroenteritis virus on the villous enterocytes of newborn pigs. J. Virol. 68, $7253-7259$ 